\title{
What can the science of well-being tell the discipline of psychiatry - and why might psychiatry listen?
}

\author{
Phil Hanlon \& Sandra Carlisle
}

\begin{abstract}
There is a field of knowledge that speaks of the promotion of positive mental health, well-being and happiness yet it may not be well-known to all psychiatric practitioners. Economists, geneticists, positive psychologists, evolutionary psychologists, neuroscientists and sociocultural researchers have all contributed to what might be termed the emerging science of well-being. This article provides a brief introduction to this complex topic. We outline some of the findings, theories and arguments from this comparatively new but burgeoning research area. We also rehearse some critical responses to this field which indicate that both the evidence on well-being and the implications for practice and policy might be less straightforward than researchers sometimes imply. We conclude by suggesting that psychiatrists, as leaders in the field of mental health, might want to consider the implications (positive and negative) of well-being research for the development of their own discipline and professional practice.
\end{abstract}

The primary goal of psychiatry has long been the relief of suffering associated with symptoms of mental disorder. Psychiatrists draw on a combination of empirical (research and/or practice-based) evidence about mental illness and its treatment, together with their own professional knowledge, on the assumption that this enables the best available treatment for people suffering from psychiatric morbidity. This is an important task and considerable unmet need still exists. At the same time, however, the wider society has become concerned about positive mental health and well-being. This may be partly because the issue of how people feel - especially their level or degree of happiness - is now taken seriously by many in the broad scientific community, with more than 3000 studies on the subject of happiness and subjective well-being being published since 1960 (Nettle, 2005). Yet this emerging field of knowledge may not necessarily be well-known to, or understood by, all psychiatric practitioners. We consider here what this new field might be able to tell the established discipline of psychiatry, and why psychiatrists, as leaders in the field of mental health, might be interested to hear and reflect on it.
The reader should bear in mind that well-being research is beset with definitional problems, despite efforts to resolve these. For example, researchers tend to use a multiplicity of terms to refer to well-being, sometimes distinguishing between them and at other times treating them as synonymous. Measurements of the various constructs of well-being are similarly fraught, with different conceptions of the term being preferred and used by different researchers and for different purposes.

As we provide only a broad-brush outline of the field (which fails to do justice to some complex and sophisticated research endeavours), interested readers are invited to consult the following texts for a fuller picture: Seligman (2002), Shah \& Marks (2004), Huppert et al (2005) and Layard (2006).

\section{Economics and 'the happiness gap'}

We need to be aware that modern economic theory evolved in a time of scarcity, when the problem was not an excess of goods but a shortage: neoclassical economic thinking therefore has little use for the

Phil Hanlon is Professor of Public Health at the University of Glasgow. His current research interests include 'culture and health', uses of integrated public health data and evaluation of complex public health interventions. Sandra Carlisle is a Research Fellow, also at the University of Glasgow (Public Health and Health Policy, Glasgow University, 1 Lilybank Gardens, Glasgow G12 8RZ, UK. Email: s.carlisle@clinmed.gla.ac.uk). She has a PhD in community health sciences and an MSc in medical social anthropology, and has conducted a wide variety of policy-relevant research and evaluation studies. Current research interests include sociocultural approaches to understanding health, well-being and inequalities, and ethnographic and theory-focused methodologies applied to research and evaluation. 
Box 1 Economics and 'the happiness gap': is life getting better or worse?

\section{Key points}

Research shows that levels of subjective well-being in high-income countries have remained static since the 1960s, and may now be declining. This is so despite rising personal incomes and improvements in living conditions for most people. In so far as traditional economic theory assumes that 'better equals more', these findings represent a paradox.

concept of 'enough' or 'more than enough'. Because research shows that, within societies, the wealthiest people are happier than the poorest, this supports the fundamental economic position that wealth is a self-evident good, increasing choice and permitting individuals to 'maximise their utility'. Yet there is also a solid body of evidence from economic research which shows that individual increases in income, once past a threshold at which basic needs are satisfied, produce diminishing returns in average levels of well-being (e.g. Easterlin, 1974). Moreover, it is known that there is little correlation between improvements in the objective conditions of social welfare and average levels of subjective well-being (Easterbrook, 2003). For example, many serious health and social problems that afflicted us in the past have been resolved, and we have seen an overall trend in economic growth over many decades (Shah \& Marks, 2004; Layard, 2006). Why, then, do people apparently feel worse? For economists these are paradoxes in need of explanation. As Nesse (2005) says, 'what we have been doing to increase general happiness is no longer working, and there is no consensus about what we should try next'.

(See Box 1 for the key points of this section.)

\section{Genetics, brain biology, evolution and culture}

A range of disciplines have contributed to the science of well-being in ways that shed light on the above problem. We summarise in the following subsections some of the evidence and arguments.

\section{Genetic influences, set-points and the potential for change}

Lykken \& Tellegen's (1996) comparison of data from a large sample of twins in the Minnesota Twin Study
Box 2 Genetic influences, set-points and the potential for change

Key points

- Our levels of well-being are significantly influenced by our genetic inheritance

- Most populations score above mid-point on measures of average levels of well-being, within a comparatively small range

- Humans adapt to most positive and negative changes in life, with a few significant exceptions

- Potential for improving well-being may lie within activities to which we do not readily adapt

found that at least $50 \%$ of the variance in individual well-being can be attributed to genetic differences. Circumstantial factors such as income, religion or marital status accounted for only $8 \%$. Research also suggests that subjective well-being across populations is subject to a form of homoeostatic control: there was comparatively little variation between populations, and well-being scores above mid-point were found to be a cross-cultural norm (Veenhoven, 2005).

Evidence indicates that people adapt to most changes, whether positive or negative, which leads some psychologists to assert that individuals have a 'set-point' for well-being from which there is little significant variance - evidence that Nesse (2005) calls 'profound and disturbing'. Yet we also know that certain events can have a far-reaching influence: for example, loss of a spouse or job has been shown to produce long-lasting damage to our well-being. Huppert (2005) also argues that the role of genetic factors can be minor when compared with environmental factors such as early nurturing. She stresses the role of intentional activities in determining one's long-term happiness level: because these are under our control and can be altered, we do not adapt to their beneficial effect.

(Key points: Box 2.)

\section{'Hardwired' humans: contributions from neuroscience and evolutionary psychology}

Neuroscientists suggest that human brain evolution has encompassed certain biological changes and predispositions that, in conjunction with a modern lifestyle, may present risk factors for well-being. The limbic (palaeo-mammalian, emotional) brain has evolved such that behaviour is based primarily 
on cognitive reasoning rather than hormonal instruction. This removal of biological restrictions to behaviour has enabled the cognitive (executive) brain to emancipate human lifestyle from the external environment and create an artificial environment that caters for and satisfies its own hedonic needs (Keverne, 2005). Moreover, because substantial brain growth in humans is postponed to the postnatal period, there are particular implications for the well-being and emotional development of young people. For example, they have to learn to manage emotions (including when and how to display them) comparatively early in life. How teenagers rate themselves and how they want to be seen by others have been found to be strong disposing factors for addiction at a time when their brain is 'insecure' (Keverne, 2005).

Evidence from brain biology is supplemented by insights from evolutionary psychology. Nesse (2005) reasons that natural selection should ensure that our baseline mood and emotional responsiveness remains 'somewhere in the vicinity of those that maximised reproductive success in the ancestral environment'. From this perspective, feelings may matter little, so long as they adjust future behaviour in ways that allocate effort efficiently. It may thus be hard to control negative emotions such as sadness or envy because those who could do this were actually deprived of crucial tools for adaptation, whereas those whose emotional experiences mapped accurately onto situations in which they were useful had a selective advantage. So, one explanation for our inability to achieve greater happiness is that our biology adapts too slowly to rapidly changing social and cultural environments: humans living in highincome countries are 'stone-agers in the fast lane'.

Theories from this discipline also suggest that humans appear biologically designed to pursue social goals that may not be conducive to their longterm well-being. Thus, we adopt the social norms and behavioural strategies of those around us that ensure reproductive success, and we 'feel good' when pursuing such strategies successfully - social status, a professional career, 'positional goods' and so on. However, because we are investing in sources of happiness that are intrinsically relative to what others have and are comparatively rare or restricted in their availability, this can result in escalating arms races in which only a few can succeed. Modern social structures induce motivational responses that leave individuals possibly sacrificing much in life in order to pursue social goals that require massive effort, even though their attainment remains uncertain. Worse still, alternatives may be few and unsatisfactory, yet psychologists note that an inability to disengage from a major but unreachable life goal is a recipe for serious depression.
Box3 'Hardwired' humans: contributions from neuroscience and evolutionary psychology

Key points

- Brain evolution has encompassed biological changes that, in conjunction with a modern lifestyle, may present risk factors for wellbeing

- Evolutionary psychology suggests that humans are endowed with a positional psychology which motivates striving and competition for goals which have recognised social value and contribute to our reproductive fitness but may not necessarily lead to longer-term contentment

Research confirms that we are not happy when those around us have more than we do. Our otherwise apparently irrational urge to stay on the 'hedonic treadmill' and practise the relentless accumulation of positional goods is thus explained by the positional psychology developed over human evolution. This may suggest an inbuilt human bias towards dissatisfaction rather than happiness (though economists, among others, would not agree with this) and that we are more predisposed, by our inbuilt competitiveness, towards unhappiness than circumstances may actually merit.

(Key points: Box 3.)

\section{Emotions: 'hardware' meets 'software'}

Researchers with an interest in the relationship between biology, culture and well-being have suggested that the picture is more complicated than thinking from genetics, neuroscience and evolutionary psychology would suggest, because the experience of emotional well-being is the combined product of biological, psychological, social and cultural factors. Confusion has arisen, in part, because psychobiologists have focused on the 'hardware' of the neurophysiological processing of emotion, whereas sociocultural researchers have focused on the 'software' of culturally variable and socially learned knowledge that mediates our emotional appraisal.

Veenhoven's (2005) research, for example, finds cross-cultural variations in happiness even across high-income countries: former communist countries are at the bottom of the 'happiness league table', and Switzerland is at the top, closely followed by the Nordic countries (Veenhoven, 2005). On a somewhat different note, although happiness is a core cultural value for people living in North America, this emphasis can be viewed as naive, verging on 
Box 4 Emotions: 'hardware' meets 'software'

Key points

- Although levels of well-being vary across cultures, general features of emotion processing (a cognitive function) are shared by all humans. However, our experience and evaluation of emotions are also mediated by cultural and social values and meanings

- Social and cultural influences (such as the nature of the goals valued in any particular society) may present threats to well-being, despite our biological predisposition to pursue those goals

infantile, by other societies across the world, such as the French or Russians, which draw on very different 'cultural scripts' (Wierzbicka, 1997).

It seems that emotions have both universal and culturally variable components. Although general features of cortical and subcortical emotional processing are pan-human, the evaluation and experience of emotion is also mediated by culture (Hinton et al, 1999). Because our biological 'hardware' is programmed by the 'software' (culture, learning, experience) available within a particular environment, there are implications for the relationship between the way we live and compromised well-being. Taken together with insights from evolutionary psychology, this suggests that average levels of wellbeing can be threatened by living in a sociocultural milieu generally characterised by competition and striving for status, even though the wealthiest are able to thrive.

(Key points: Box 4.)

\section{The psychology of well-being}

We now turn to the psychological question of what makes life worth living. Layard (2006) cites evidence from a Texan survey of more than 900 women: essentially, sex and socialising seem to be the activities producing the most happiness, whereas child care, housework and spending time with a spouse or partner come further down the list. However, these findings relate to feelings measured over the short term, which do not necessarily tell us much about what makes for long-term wellbeing over the life course. The 'Big Seven' factors affecting longer-term happiness, according to the US General Social Survey quoted by Layard, are family relationships, financial situation, work, community and friends, health, personal freedom, and personal values.
Psychologists therefore tend to distinguish between three levels or types of subjective well-being:

1 transient feelings of enjoyment: these feelings are measurable both in terms of personal report and brain activity;

2 evaluativejudgements about the balance of our feelings over time: these are used to measure an individual's overall life satisfaction;

3 the experience of a state of flourishing and fulfilment of one's potential, sometimes known as 'flow' or engagement.

In levels / types 1 and 2, psychologists are drawing a distinction between 'the experiencing self' and 'the remembering self'. Some (e.g. Kahneman et al, 1999) believe that those studying happiness should pay attention to people's actual experiences, rather than just their reflections, especially if research is to inform policy. Others (e.g. Seligman, 2002) focus on the remembering self, arguing that experiences are too transient to provide an accurate picture of well-being as life satisfaction. However, research has also shown that such assessments are notoriously sensitive to context and at least partly dependent on mood (Schwarz \& Strack, 1999: p. 62). We are, apparently, pretty unreliable as witnesses to and judges of the state of our own lives, to the extent that we are more prone to make positive evaluations when the sun shines. We should also note that, although the first two types/levels are the province of individual judgements, the third is open to moral judgement by others.

Huppert et al (2005) construe well-being as 'life going well, characterised by health and vitality, by happiness, creativity and fulfilment'. They also stress that well-being is more than pleasant emotions because it must also encompass human resilience - the ability to thrive in the face of adversity. Echoing Nesse (2005), they suggest that positive emotions are not enough and negative emotions are sometimes necessary. However, in the same volume, Fredrickson (2005) prefers to focus solely on positive emotions. Her 'broaden-and-build theory of positive emotions', based on research with groups rather than individuals, proposes that the frequent experience of positive emotions broadens cognitive processes and builds enduring coping resources, leading to resilience. Participating in activities and engaging with one's environment are stressed as important.

An overall message from many in this field of research is something along the lines of 'adjust your goals to what is possible, be satisfied with who you are and what you have, and happiness will probably follow'. This is usually described as 'wise advice with an increasingly strong scientific foundation', because happiness is viewed not as a reachable goal but as a state that emerges when an 


\section{Box 5 The psychology of well-being}

Key points

- Measures of well-being may focus on our experiences or our memories, depending on whether researchers are investigating what makes people happy over the short term, or a lifetime, or what constitutes a good life

- The fullest definitions of well-being pay attention to the need for resilience in the face of adversity

- Advice for happiness tends to be based on adjusting one's goals to what appears possible, and acceptance of both self and life circumstances rather than endless striving

individual is making good progress towards life goals (Nesse 2005).

(Key points: Box 5.)

\section{Contributions from positive psychology}

Positive psychology is an umbrella term for the study of positive emotions, positive character traits and enabling institutions (Seligman \& Csikszentmihalyi, 2000). It arose out of psychologist Martin Seligman's campaign to shift the emphasis of his discipline away from its traditional focus on the negatives of mental health and well-being. Positive psychologists claim that happy people are healthier, more socially engaged and more successful (Seligman, 2002). Exponents of the positive psychology movement stress that they use the term 'happiness' to refer to the combination of positive emotion, engagement and meaning in life.

The cultivation and practice of certain character strengths is integral to positive psychology's approach. These contribute to the fulfilment of what Seligman et al (2005a) call 'strengths of the heart', i.e. hope, love, gratitude and zest, because of their demonstrated association with life satisfaction. Although concepts such as the pleasant life and the good life are valued, some positive psychologists stress the greater importance of the meaningful life - a life devoted to fulfilling one's potential, often in the service of others (Seligman, 2002).

A range of interventions for improving wellbeing have been tested by the exponents of this new movement. There is evidence (Huppert, 2005; Layard, 2006) from randomised controlled trials that cognitive-behavioural therapy is effective at reducing the impact of negative emotions. 'Pleasant activity training' enables us to discern what activities really make us happy and then practise them, thus increasing our stock and experience of positive

\section{Box 6 Contributions from positive psy-} chology

Key points

- Positive psychology combines the study of positive emotions, positive character traits and enabling institutions

- A range of interventions have provided evidence of effectiveness in improving individuals' subjective well-being

- People are encouraged to identify and practice their particular signature strengths as a way of enhancing meaning in life.

- Widespread social interventions to promote well-being are also advocated emotions. Mindfulness meditation has been shown effective in enhancing subjective well-being across a range of populations: training involves focusing, through meditation, on what is taking place in the moment in one's internal and external environment. The goal is partly to enable people to reframe their problems by focusing on a wider set of concerns. Other individual-level interventions found effective in trials by Seligman et al (2005a) include: listing three good things that happen every day for a period of some weeks; making gratitude visits to significant mentors; and practising one's signature strengths. Other psychologists also suggest that interventions could take place through education, health promotion and the media, and 'early-years' interventions and positive parenting training.

(Key points: Box 6.)

\section{Some critical responses}

Reservations about the new focus on well-being, the validity of its research and its policy implications exist. For example, some psychologists remain sceptical that personality is readily flexible, that individuals should change existing coping styles that work for them, and that a positive attitude can solve complex human problems (Held, 2005). And as a reviewer of this article pointed out, it is unclear whether the kinds of 'well-being exercises' advocated by positive psychologists, among others, can really succeed in 'keeping the psychiatrist away'.

The question of whether life is getting better or worse also appears to involve multiple and potentially conflicting and contradictory types of evidence. Eckersley (2004) points out that claims from the science of well-being are often based on statistical correlations rather than causal connections. The direction of causality can be unclear. Measures of subjective well-being tend to emphasise happiness, 
but this can be linked to positive self-illusions, which suggests that subjective well-being may involve a degree of self-deception, excessive optimism and an overestimation of personal control over life. There are also problems with research based on survey evidence, because questions most commonly used to assess levels of well-being conflate the subjective assessment of objective well-being with the objective assessment of subjective well-being (O'Neill, 2006). In addition there is the inescapable fact that, as happiness is inherently subjective, surveys of well-being that are unable to examine the context of individual lives miss much that would otherwise be of value.

A related point is that research into well-being and happiness can be influenced by the assumptions of its researchers. Let us consider, as illustration, the 'top ten' countries found in two different global surveys (Table 1).

\section{The happiness top ten}

In White's (2007) global projection of subjective well-being (a 'map of global happiness'), which draws on data from Marks et al (2006), the top ten are mainly European countries. However, in Marks et al's own ranking, using a measure called the Happy Planet Index, although the top country is a Pacific island, the list is dominated by smaller Latin American countries. There is clearly a marked difference between the two sets of findings. In reality, happiness and subjective well-being are only one of the components measured in the two surveys. White et al also consider access to education, life expectancy and gross domestic product in their calculations, whereas Marks et al's ranking is influenced by measurement of countries' ecological footprint. The

\begin{tabular}{|c|c|c|}
\hline \multirow[b]{2}{*}{ Rank } & \multicolumn{2}{|c|}{ Source } \\
\hline & Marks et al (2006) & White (2007) \\
\hline 1 & Vanuatu & Denmark \\
\hline 2 & Colombia & Switzerland \\
\hline 3 & Costa Rica & Austria \\
\hline 4 & Dominica & Iceland \\
\hline 5 & Panama & The Bahamas \\
\hline 6 & Cuba & Finland \\
\hline 7 & Honduras & Sweden \\
\hline 8 & Guatemala & Bhutan \\
\hline 9 & El Salvador & Brunei \\
\hline 10 & $\begin{array}{l}\text { Saint Vincent and } \\
\text { the Grenadines }\end{array}$ & Canada \\
\hline
\end{tabular}

latter's approach may be based on sound reasons in terms of broader global well-being, but it means that countries with high levels of consumption are inevitably downgraded in their list.

It has been pointed out that the contemporary focus on happiness in the West is consonant with North American cultural values (Wierzbicka, 1997) but may fit poorly with a more international perspective on what constitutes good mental health and emotional well-being (Kitayama \& Marcus, 1997). A related point is the serious charge that the current preoccupation with happiness is primarily a self-indulgent concern of prosperous and comfortable people, living in the more wealthy areas of the globe. White (2007) has pointed out that the commercial development of positive psychology is taking place in those countries where subjective well-being is highest, which leaves positive psychology open to the accusation of selling self-help to the 'worried well'. Can we really speak of well-being and happiness in relation to those parts of the world where daily life itself remains a precarious matter? As some of the poorest and/ or most troubled countries in the world lie at the 'bottom' of both the Happy Planet Index listing and the 'map of global happiness', attention to well-being can be justified.

\section{The politics of well-being}

What we might call 'the politics of well-being' appear somewhat muddled, with recommendations being made that range from increasing individual happiness to a focus on leading worthwhile, fulfilling lives. In the latter case, we might ask who should be the judge of what constitutes the 'full' rather than 'empty' life, advocated by Seligman (2002)? Nor can we assume that what appears to make people happy at the individual level is easily transferable into public policy, though such recommendations are found in the literature. The increasing interest in well-being found within public and media discourse, areas where some research has been successfully popularised, merits attention because various moral and political ideologies seem to be emerging in the guise of self-evident, universal human 'goods' and / or recommendations for social policy (e.g. Shah \& Marks, 2004). These include restricting social mobility, in order to promote family and community cohesion, and earnings capacity, in order to avoid promoting social envy and competition (Layard, 2006).

More generally, critics such as Williams (2000) suggest that we are witnessing 'creeping forms of mental disorder', including a range of emotional states that may not elicit a diagnosis of mental illness yet are viewed as needing help/remediation in one form or another. This therapeutic culture of the self offers 'technologies for the government of the soul' 


\section{Box 7 Some critical responses}

Key points

- The science of well-being is still undergoing development so some of its conclusions may be less sound than exponents of the field might claim

- Critics are sceptical of 'happiness' as a universal and self-evident human good which can and should provide a valid focus for social policy

- We may be witnessing the emerging of a therapy culture which extends beyond the disciplines of psychiatry and psychology. Therapy culture conforms to contemporary ideals of self-regulation yet may militate against people's sense of their own abilities to cope with adversity

which are consonant with contemporary political/ economic principles and moral ideas. Evidence of this trend towards the compulsion to be happy is readily found in the Western media. Williams argues that the emphasis on personal happiness and fulfilment found across the range of such therapies is based on illusions of personal growth and fulfilment that are never quite achievable, yet provide the comfort of enabling people to take responsibility for their lives and satisfy their needs. Williams even suggests that prevailing discourses of happiness and the good life may contribute to breeding unhappiness because of unrealistic expectations.

(Key points: Box 7.)

\section{Implications for psychiatry?}

Most well-being researchers view their field as a complement to, rather than a replacement for, the psychiatrist's goal of easing misery and treating morbidity. Notwithstanding the caveats outlined above (and others that may occur to our readers), psychiatrists - as leaders in the field of mental health - might approve of this new science's goal of contributing to a more complete understanding of human well-being because of its potential contribution to ameliorating mental suffering. Huppert (2005), for example, suggests that if the mean of positive mental health in the population could be shifted by universal interventions, the small shift in the average would actually mean a substantial decrease in the numbers of people currently languishing.

We suggest that psychiatrists might want to consider and discuss the implications (positive and negative) of well-being research for the development of their own discipline and professional practice. The fact that this field is now a recognised scientific endeavour, providing a steadily expanding evidence base of 'what works' in improving individual wellbeing, suggests that such attention is warranted, even if uncritical acceptance is not. The focus on recommendations for social and economic policies is also of interest, though questions remain over their general feasibility.

\section{Acknowledgements}

We are indebted to the helpful and knowledgeable comments made by a reviewer of this article. Its flaws remain our own.

\section{Declaration of interest}

This article forms part of a research project funded by the National Programme for Improving Mental Health and Well-being in Scotland.

\section{References}

Easterbrook, G. (2003) The Progress Paradox: How Life Gets Better while People Feel Worse. Random House.

Easterlin, R. A. (1974) Does economic growth improve the human lot? In Nations and Households in Economic Growth: Essays in Honor of Moses Abramovitz (eds P. A. David \& M. W. Reeder), pp. 89-126. Academic Press.

Eckersley, R. (2004) Well E Good: How We Feel and Why It Matters. Text Publishing.

Frederickson, B. L. (2005) The broaden-and-build theory of positive emotions. In The Science of Well-Being (eds F. A. Huppert, N. Baylis \& B. Keverne), pp. 217-240. Oxford University Press.

Held, B. S. (2005) The "virtues" of positive psychology. Journal of Theoretical and Philosophical Psychology, 25, 1-34.

Hinton, A.L. (ed.) (1999) Biocultural Approaches to the Emotions. Cambridge University Press.

Huppert, F. A. (2005) Positive mental health in individuals and populations. In The Science of Well-Being (eds F. A. Huppert, N. Baylis \& B. Keverne), pp. 307-340. Oxford University Press.

Huppert, F. A., Baylis, N., Keverne, B. (eds) (2005) The Science of Well-Being. Oxford University Press.

Kahneman, D., Diener, E. \& Schwarz, N. (eds) (1999) WellBeing: The Foundations of Hedonic Psychology. Russell Sage Foundation.

Keverne, E. B. (2005) Understanding well-being in the evolutionary context of brain development. In The Science of Well-Being (eds F. A. Huppert, N. Baylis \& B. Keverne), pp. 35-56. Oxford University Press.

Kitayama, S. \& Markus, H. R. (eds) (1997) Emotion and Culture: Empirical Studies of Mutual Influence. American Psychological Association.

Layard, R. (2006) Happiness: Lessons from a New Science. Penguin Books.

Lykken, D. \& Tellegen, A. (1996) Happiness is a Stochastic Phenomenon. Psychological Science, 7, 186-189.

Marks, N., Abdallah, A., Simms, A., et al (2006) The (un)Happy Planet Index. An Index of Human Wellbeing and Environmental Impact. nef (the new economics foundation).

Nesse, R. M. (2005) Natural section and the elusiveness of happiness. In The Science of Well-Being (eds F. A. Huppert, N. Baylis \& B. Keverne), pp. 3-32. Oxford University Press.

Nettle, D. (2005) Happiness: The Science Behind Your Smile. Oxford University Press. 
O'Neill, J. (2006) Feature review. New Political Economy, 11, $447-450$.

Schwarz, N. \& Strack, F. (1999) Reports of subjective well-being: judgmental processes and their methodological implications. In Well-Being: The Foundations of Hedonic Psychology (eds D. Kahneman, E. Diener \& N. Schwarz), pp. 61-84. Russell Sage Foundation.

Seligman, M. E. P. (2002) Authentic Happiness. Free Press.

Seligman, M. E. P. \& Csikszentmihalyi, M. (2000) Positive psychology: an introduction. American Psychologist, 55, 5-14.

Seligman, M. E. P., Steen, A., Park, N., et al (2005a) Positive psychology progress: empirical validation of interventions. American Psychologist, 60, 410-421.

Shah, H. \& Marks, N. (2004) A Well-Being Manifesto for a Flourishing Society. nef (the new economics foundation).

Veenhoven, R. (2005) World Database of Happiness, Distributional Findings in Nations. Erasmus University (http:/ / www.world databaseofhappiness.eur.nl)

White, A. (2007) A global projection of well-being: a challenge to positive psychology? Psychtalk, 56, 17-20 (http:/ /www.le.ac. uk/pc/aw57/world/sample.html).

Wierzbicka, A. (1997) Emotion, language, and cultural scripts. In Emotion and Culture: Empirical Studies of Mutual Influence (eds S. Kitayama \& R. H. Markus), pp. 133-196. American Psychological Association.

Williams, S. J. (2000) Reason, emotion and embodiment: is 'mental' health a contradiction in terms? Sociology of Health and Illness, 22, 559-581.

\section{MCQs}

1 The concept of well-being:

a has historically been the central concern of psychiatry

b has become intensively studied only relatively recently

c is a straightforward and non-contested construct

$\mathrm{d}$ is exactly equivalent as a concept to positive mental health

e cannot be measured - either in individuals or in populations

2 In modern, Western-style societies, measures of subjective well-being among the population have:

a increased in parallel with rising wealth since the Second World War

b declined with rising wealth in an inverse relationship

c are unaffected by measure of national wealth such as GDP

$\mathrm{d}$ have not been measured because of technical difficulties

e ceased to rise in parallel with GDP in the mid-1970s and are now static or declining despite continuing economic growth.

3 Levels of well-being in individuals:

a show little evidence of genetic influences

b are accounted for ( $80 \%$ of variance) by circumstantial factors such as income, religion or marital status c tend towards pessimism, with most reporting low levels of well-being

d show that individuals tend to adapt to most changes, whether positive or negative

e cannot be influenced at all by intentional activities.

4 Evidence from evolutionary psychology suggests that:

a our baseline mood and emotional responsiveness remains close to that which maximised reproductive success in the ancestral environment

b it should be easy for us to control negative emotions such as sadness or envy

c we have adapted many new emotional responses to suit our modern environment

d humans appear biologically designed to pursue happiness in preference to social goals, which may not be conducive to their long-term well-being

e we make up our own minds about what is important without much reference to the rest of society.

5 Our understanding of 'what makes life worth living' includes:

a evidence that child care, housework and spending time with a spouse or partner seem to be the activities producing the most happiness, whereas sex and socialising come further down the list

$\mathrm{b}$ the insight that a state of flourishing and fulfilment of one's potential, sometimes known as 'flow' or engagement, is very important

c that people's actual experiences do not matter - the only thing that is important is our reflections on experiences

d we are very reliable as witnesses to and judges of the state of our own lives

e positive emotions have no important interaction with cognitive processes. 